\title{
Reviewers for Volume 13
}

We would like to thank the following reviewers for their contribution to Volume 13 of $A L T-\mathcal{F}$ :

Graham Alsop

Russel Beale

Hugh Davis

Gordon Doughty

Graham Gibbs

Janet Hanson

Kim Isroff

Ann Jones

Bernard Lisewski

Patrick McAndrew

Colleen McKenna

Nora Mogey

John O'Donoghue

Paul Riddy

Malcolm Ryan

Kerry Shepherd

Rob Ward

Denise Whitelock
Philip Barker

John Cook

Marten De Laat

Karen Fill

Stephen Greenwood

Peter Hartley

Jill Jameson

Chris Jones

Phil Levy

Claire McAvinia

Carmel McNaught

Beverley Oliver

Caroline Pelletier

George Roberts

Niall Sclater

Janice Smith

Adam Warren

It is a convention in many journals to publish acceptance rates as an indication of the journal's quality, but this is an approach that sits uneasily with our aims. As editors, our approach is to support authors in developing and improving their submissions to $A L T-\mathcal{f}$ through a constructive, iterative process; simple statistics about rejection rates undercut this intention. Consequently, we will provide a fuller picture of the reviewing process.

Our reviewers are vital to our efforts at dialogue with authors, providing detailed and considered feedback. This is reflected in our submission statistics, where only $15 \%$ of submissions to $A L T-7$ in 2005 were accepted subject to minor amendments. Reviewers recommended that a further $35 \%$ of submissions in 2005 were suitable for publication after major amendments; of these submissions, $70 \%$ were eventually 
published. This gives a total acceptance rate of about $40 \%$, but shows the value of the reviewing process in raising the quality of the majority of articles we publish.

Collectively, our reviewers have contributed to enhancing the quality of $A L T-\mathcal{F}$, and we are extremely grateful to them.

Jane Seale, Martin Oliver and Grainne Conole

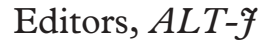

